\title{
Landmine Characterization Applying GPR Assessment and Modeling Approaches
}

Mahmoud A. Mohana*, Shereen M. Ebrahim, Abbas M. Abbas, Khamis K Mansour and Hany S Mesbah

National Research Institute of Astronomy and Geophysics, Egypt

\begin{abstract}
There are more than 119 million mines were buried in 71 countries in the world. The number of mine victims is greater than the number of the victims of nuclear and chemical weapons together. Egypt is one of the countries that suffer from the presence of landmines in its soil. Hence, around 21 million landmines are found in several locations, especially at El-Alameen and Sinai Peninsula.
\end{abstract}

Ground Penetrating Radar (GPR) is a near-surface geophysical imaging technique used for subsurface geologic, engineering and environmental investigations. It is an efficient tool for landmines detection, especially non-metal types such as PMN-2 landmine as well as its far detection capability.

In this paper, our main objective is to validate the ability of Ground penetrating Radar to discriminate between various buried targets. Wavelets transform was used to obtain the spectrum distribution of every buried target. The difference between the distributions of the different target spectrum can be considered as a finger print for each one, the summations of powers at the locations of targets were calculated and compared.

Simulation models for different targets were made. The reflections of targets were analyzed by Daubechies Wavelets (db2) transform to differentiate between different targets and to get finger print for every target. This technique was applied for field measurement of different target type and the technique revealed that the variation of finger print for every buried target. We believe that with this $\mathrm{t}$

Keywords: Ground penetrating radar (GPR); Wavelet transform; Landmine; Mine-like; Plane wave source

\section{Introduction}

Landmine clearance is a critical problem faced by many countries around the world, and the situation can be compounded by natural disasters or land development. Therefore, it is an urgent issue to detect landmines in the ground and remove them safely. For safe detection, non-touch-based detection methods are required. These methods involve the detection of landmines from the signals obtained by nontouch-based sensors, such as metal detectors and radars. Among those sensors, ground penetrating radars, or GPRs, is an attractive choice for landmine detection due to their advantages over other sensors. The GPR can be used as a stand-alone sensor or as a complementary sensor to a metal detector $[1,2]$. It can detect both metal and nonmetal landmines. Moreover, its weight can be made light, so that it can be installed in a handheld system or in a vehicle-mounted system in the form of an array of multiple antenna elements [3]. Identification of landmine from other targets or clutters is a vital task. For that reason, Wide varieties of signal processing techniques have been evolved and used to process GPR signals. Gader and Nelson et al. $[4,5]$ have proposed a gradientbased method for landmine detection. Three features based on Singular Value Decomposition (SVD), Discrete Fourier transform DFT and Principal Component Analysis, (PCA), are extracted to each signal for landmine detection, which is performed by using the Mahalanobis distance method.

Egypt is one of the most contaminated landmine countries. The problem of landmines in Egypt has started up since the World War II in the northern part if western desert. The military operations carried out by the Allied Forces and the Axis Power from 1941-1943 left varieties of about 22 Million landmines and UXO in western desert nearly along the coast of the Mediterranean sea. Not only thousands of civilians killed and injured each year, but also the social, economic and environmental impacts of those mines are disgraceful [6]. Due to the risk to implement the research of the mines in the contaminated areas, it is often carried out measurements on test sites, or laboratory studies.

In this paper, the finger prints of various buried targets of different material were obtained through the application of our technique [7]. Initially, simulation models for different targets such as perfect electric conductor (PEC), plastic (PVC) and limestone were designed by using a plane wave electromagnetic source to find guideline results for comparison in case of real field measurements.

Secondly, our technique was applied to the test site measurements (GPR data). Our MATLAB code was designed to process the data which includes the removing shift in time, the removing the antenna coupling and the automatic gain control (AGC) to improve and enhance the data. Also the moving average and the bandpass filtering, processing were used to smooth and remove unwanted frequencies before using our technique. Subsequently, we obtained the finger print of various buried targets.

\section{Methodology}

The reflected power was compared in the illustrated technique to determine the locations of targets. The continuous wavelet transform was applied to offer very good time and frequency localization. As a

*Corresponding author: Mahmoud A. Mohana, National Research Institute of Astronomy and Geophysics, Egypt, Tel: +20225543 111; E-mail: Mahamoud2746@ hotmail.com; Sherinmohamed2001@yahoo.com

Received April 24, 2015; Accepted July 08, 2015; Published July 16, 2015

Citation: Mohana MA, Ebrahim SM, Abbas AM, Mansour KK, Mesbah HS (2015) Landmine Characterization Applying GPR Assessment and Modeling Approaches. J Remote Sensing \& GIS 4: 150. doi:10.4172/2469-4134.1000150

Copyright: (c) 2015 Mohana MA, et al. This is an open-access article distributed under the terms of the Creative Commons Attribution License, which permits unrestricted use, distribution, and reproduction in any medium, provided the original author and source are credited. 
fact that the summation of reflected power from metallic targets is the maximum between all targets, so that the reflected power distribution at the target locations was studied. We found that a significant difference between power distributions of the different target spectrum, which varies depending on the target type that can be considered as a finger print for every target. Also the multiple received reflections were varied depending on target kind. This technique enabled us to discriminate between different targets.

\section{A commercial software CST [8]}

Microwave Studio program was used as a FDTD simulator for observing the electromagnetic scattering from a determined landmine (metal or plastic). Table 1 shows the numerical simulation parameters for different landmines models and limestone model as shown in Figure 1 in which plane wave is used as electromagnetic source. These models were simulated with operating frequency from $400 \mathrm{MHz}$ up to $1400 \mathrm{MHz}$; central frequency $900 \mathrm{MHz}$. The plane wave source was used instead of antennas for well describing and well controlling the electromagnetic radiation or wave from targets. It facilitates the simulation of an incident wave from a source located a large distance from the observed object in combination with far field monitors.

For the FDTD numerical simulation, exciting the model with a plane wave source requires several initial conditions to be satisfied such as:

1. 1. The surrounding space should consist of a homogenous material distribution

2. 2. Boundary conditions must be defined as the direction of incidence

3. 3. Other excitation ports must not be located on boundary conditions [9].

Figure 2a-2c illustrates the reflected signals from metal (PEC) plastic (PVC) and limestone targets respectively. In Figure 2, there were three various reflections. The first one was the direct coupling, the second one was the ground reflection and the last one was the reflection from different targets. By using the fact that the reflection of the metal target is much greater than the other reflections, then by comparing the reflected power of all targets as shown in Figure 3, we were able to confirm the location of the targets which indicates the accuracy of our results. By applying our technique on these signals, the finger print of each previous target was detected. By comparing Figure $4 \mathrm{a}-4 \mathrm{c}$ at the same location determined in Figure 3, we have found that there are different signal image for each target. The significant difference in the signal image was considered as a finger print to this specific target. By calculating the relative summation of power distribution at target locations, we found that the distribution of power from the metallic target was five times, limestone target and it was eighty double the plastic target which enables us to easily differentiate between them.

\begin{tabular}{|c|c|}
\hline Model size & $1 \mathrm{~m} \times 1 \mathrm{~m} \times 1.9 \mathrm{~m}$ \\
\hline Source & Gaussian pulse \\
\hline EM incident type & Plane wave \\
\hline Frequency range & $400 \mathrm{MHz}-1400 \mathrm{MHz}$ \\
\hline Permittivity of medium & Soil medium 4 \\
& PEC \\
PVC disk 2.7 \\
Absorbing boundary condition & $\mathrm{PML}$ \\
\hline Dimensions of disks & $16 \mathrm{~cm}$ radius $\times 7 \mathrm{~cm}$ height \\
\hline
\end{tabular}

Table 1: The numerical simulation parameters.

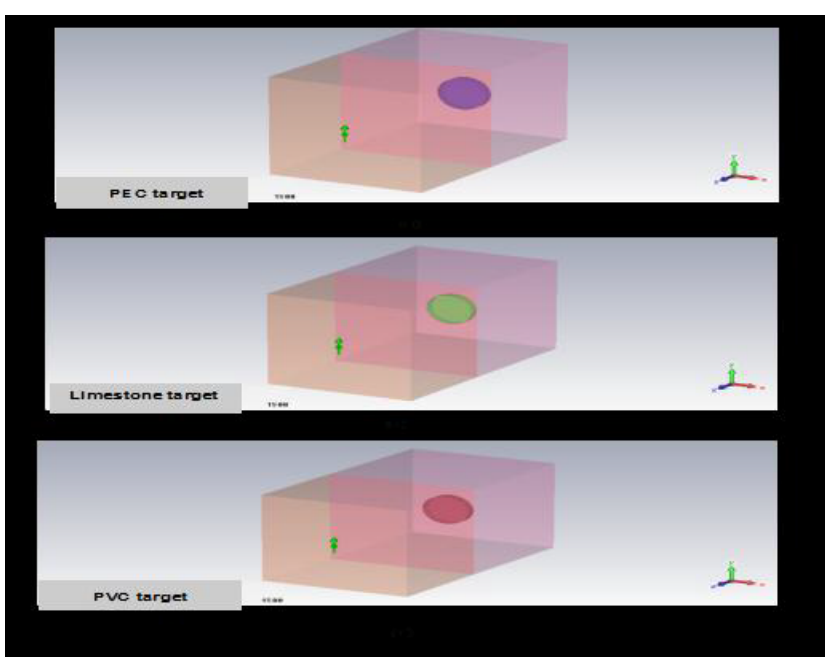

Figure 1: Plane wave models in CST program (a) PEC model (b) Limestone model (c) PVC model.
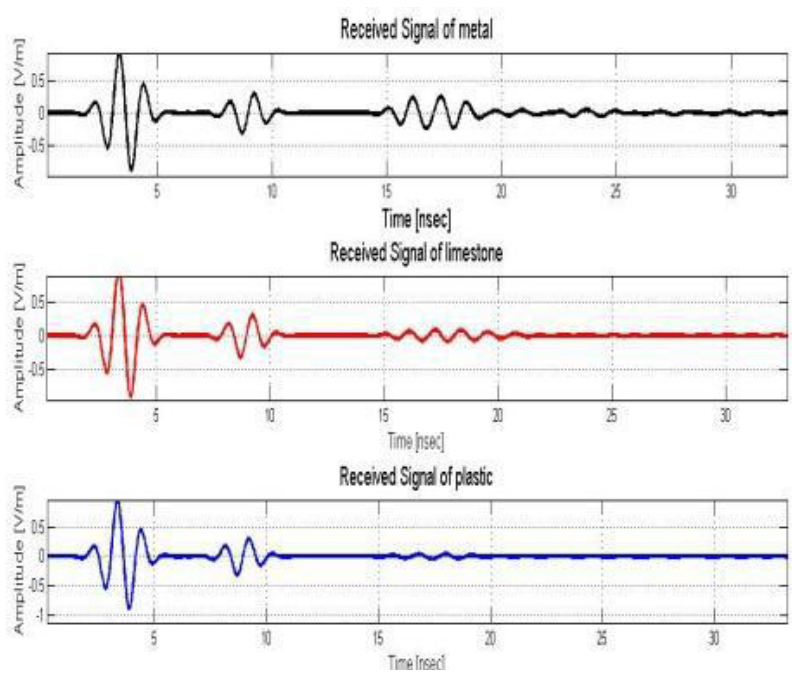

Figure 2: Reflected signal from different Targets at plane wave source (a) PEC model (b) Limestone model (c) PVC model.

Also the summation of the multiple received reflections power was varied according to targets kind as shown in Figure 5a-5c which is used as a way to distinguish between the various targets too.

For further illustration, the reflected signal from each target was extracted separately by applying time gate of the Hammining window as shown in Figure $6 a-6 c$. The finger prints of each target after using Hamming window were obtained by using the previous technique as shown in Figure 7a-7c.

\section{Test-site measurements}

An experimental test site has been constructed within the campus of the National Research Institute of Astronomy and Geophysics (NRIAG) in the Fayoum as described in The experimental test site has been surveyed by using the GPR instrument of Geophysical Survey System Inc. (GSSI) model SIR $20 \mathrm{H}$ attached to the antenna of $1.5 \mathrm{GHz}$ central frequency. 


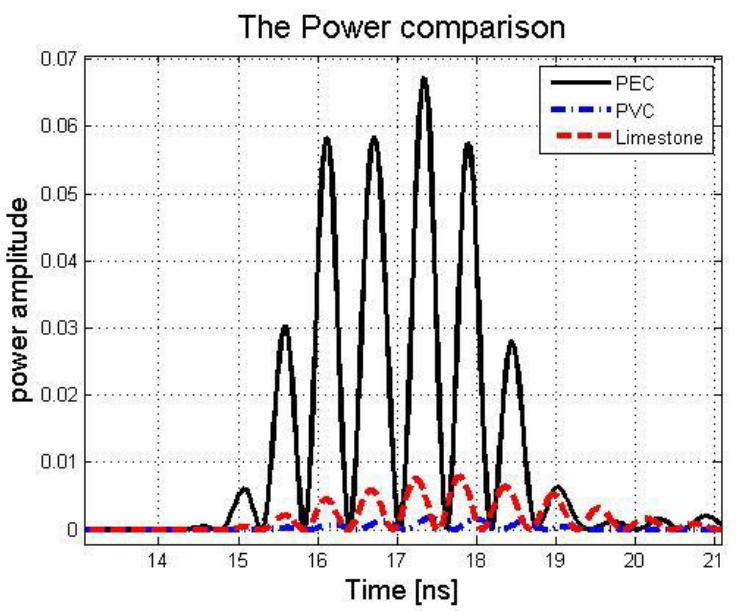

Figure 3: Reflected power comparison.

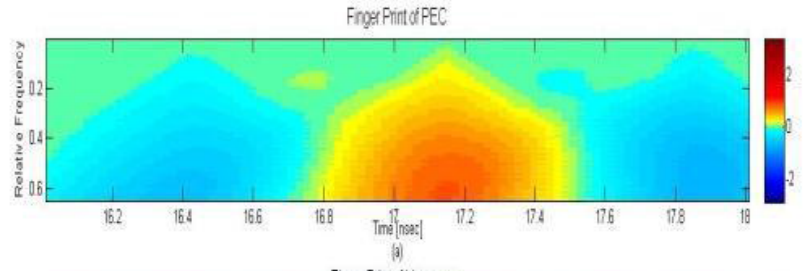

Fings Printol limestione

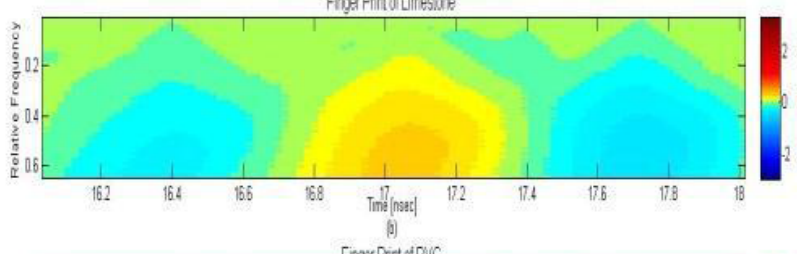

Fings Prituif PVO

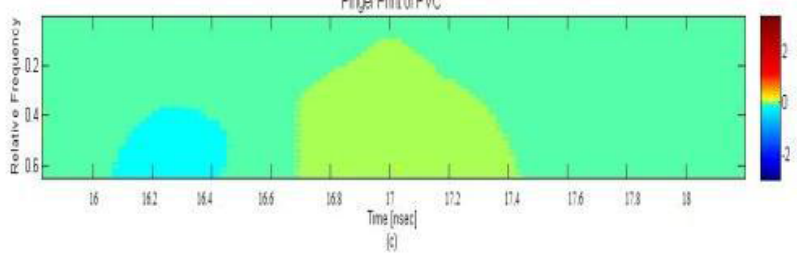

Figure 4: The finger print of targets (a) PEC (b) Limestone (c) PVC.

The one of the profiles which was carried out on a test site, that contains a set of targets made of different materials was selected as illustrated in Table 2. Figure 8 shows the GPR section of this profile. Initially, we needed to apply processing methods on the GPR data of the experimental test site. Our MATLAB code was designed to process the GPR data which included the static correction of time shift, background removal and adding the automatic gain control (AGC) to improve and enhance the data. Also the moving average and the bandpass filtering, processing were used to smooth and to remove unwanted frequencies. Figure 9 shows the final GPR section after processing.

Secondly, We applied our technique to that profile after processing. By using the reflected signal of each target, the different finger prints of real field measurements were obtained. Figure 10a-10c, show the fingerprint of each buried targets. Also by studying the summation of power distribution, we found a great ability to differentiate between

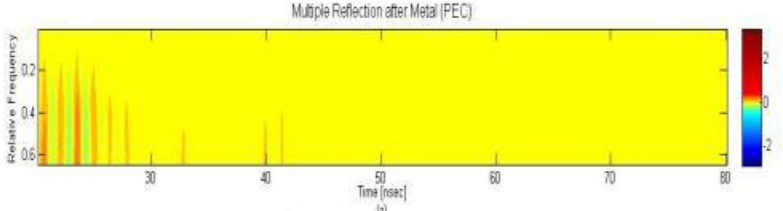

Mutple Refiection atter Limestcone
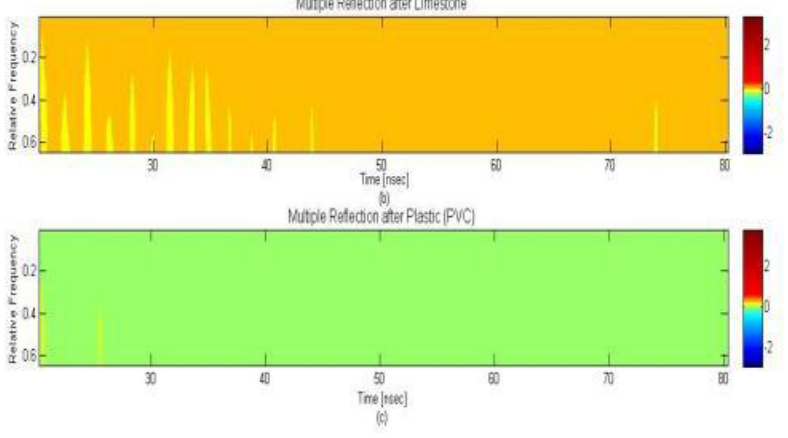

Figure 5: Multiple received reflections after targets (a) PEC (b) Limestone (c) PVC.
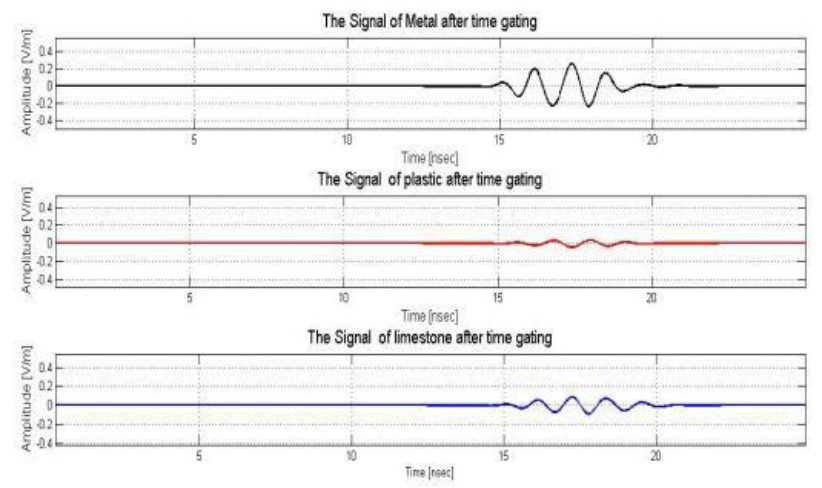

Figure 6: Reflected signal after Time gate using Hamming window (a) PEC model (b) Limestone model (c) PVC model.
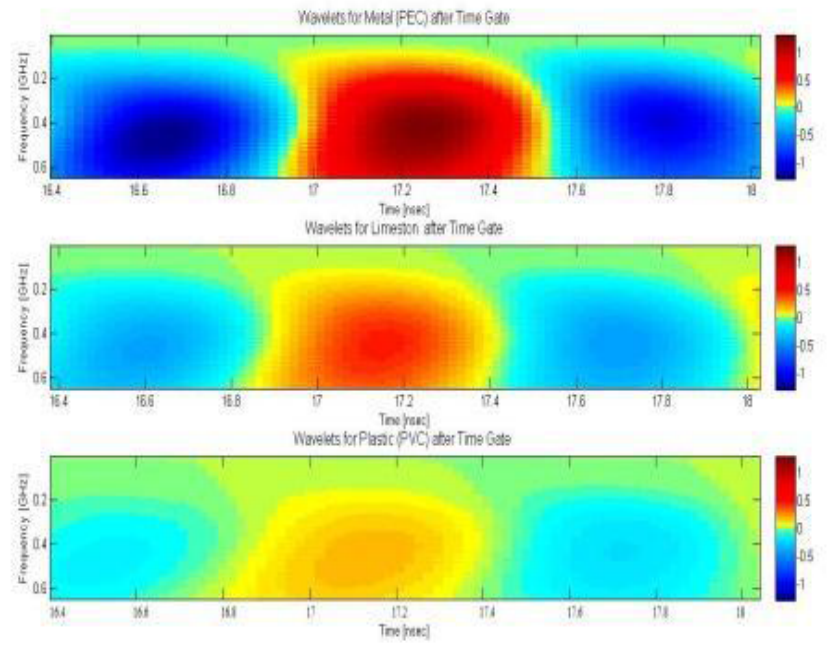

Figure 7: The finger print after time gate using Hamming window (a) PEC model (b) Limestone model (c) PVC model. 


\begin{tabular}{|c|c|c|c|c|}
\hline No & Targets & Length(cm) & Width (cm) & Depth (cm) \\
\hline 1 & Aluminum pan & & & 14 \\
\hline 2 & Computer mouse & & & 25 \\
\hline 3 & Iron disk & 25 & 3 & 25 \\
\hline 4 & Iron plate & 181.5 & 155 & 71 \\
\hline
\end{tabular}

Table 2: The parameters of the buried targets in test site after (Abbas et al. [9]).

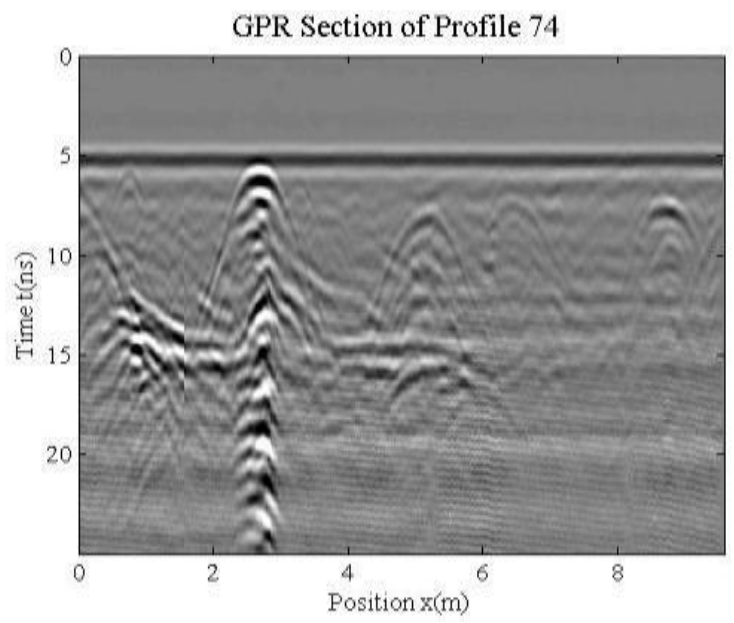

Figure 8: The GPR section.

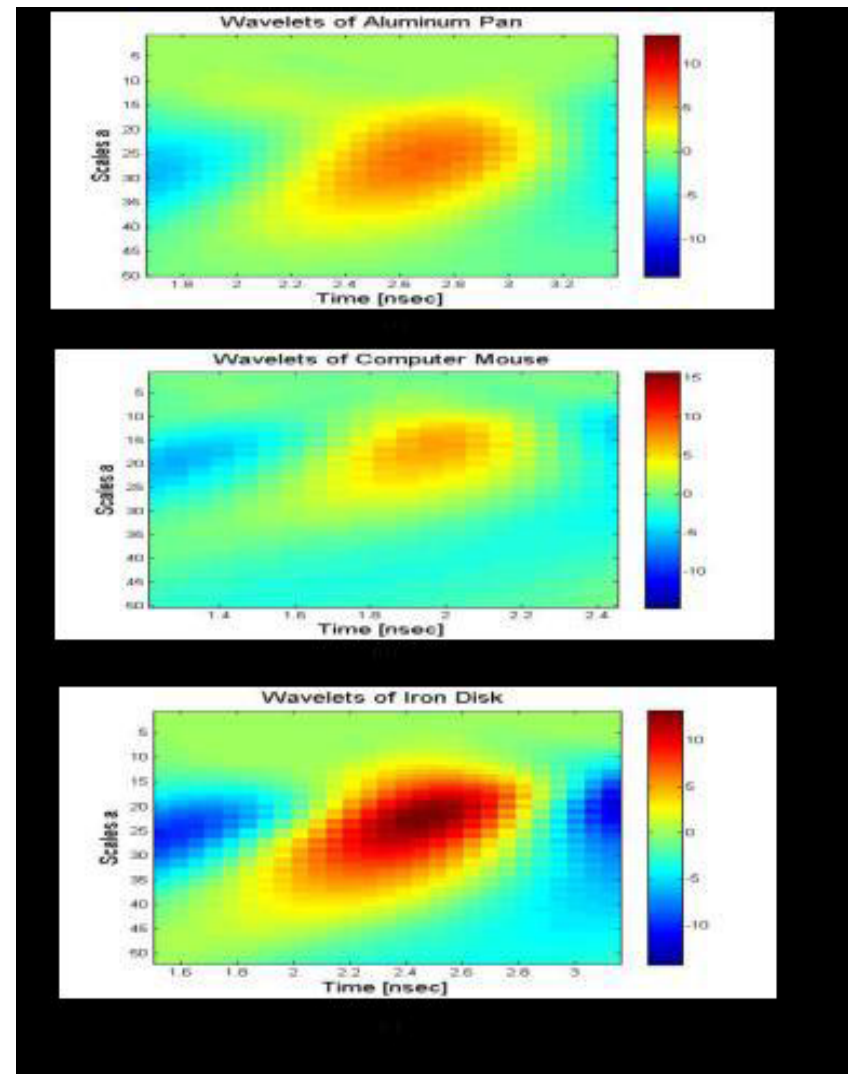

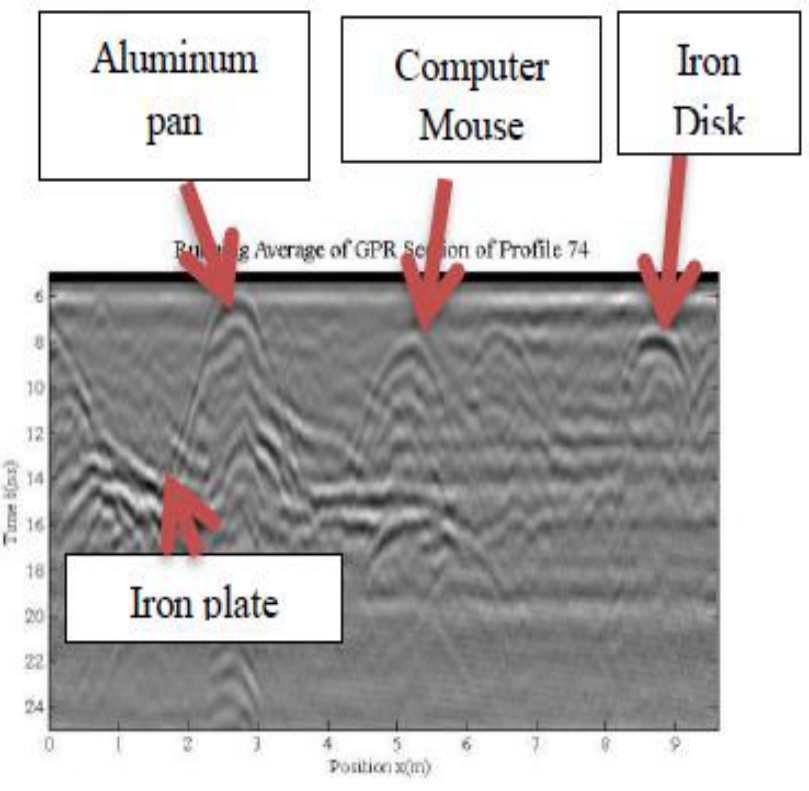

Figure 9: GPR section after running average.

the various targets where the percentages of relative summation vary in iron and aluminum and plastic.

Figure 11a-11c shows another method to discriminate between the targets by calculating the sum of multiple received reflections which varied according the target type too. The results of real measurements were very close to the results of simulation models, which confirm the credibility of our own technique.
Figure 10: The finger print of targets (a) Aluminum pan (b) Computer mouse (c) Iron Disk.

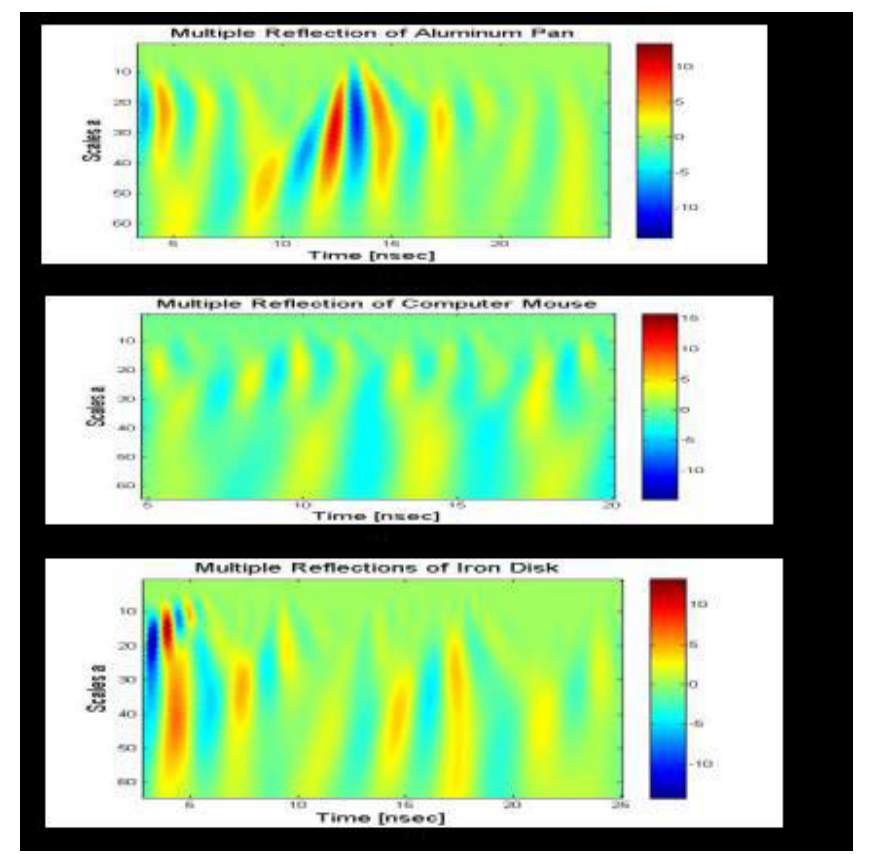

Figure 11: The multiple received reflection of targets (a) Aluminum pan (b) Computer mouse (c) Iron Disk. 


\section{Conclusion}

Many of researches were provided for the detection of landmines sites to save human life and to obtain optimum utilization of the contaminated areas. Given the gravity to implement the researches of the mines in the affected areas, it is often carried out measurements on test sites, or laboratory studies. In this paper, a novel method for landmine detection is presented. We were able to differentiate between the various targets using our own technique, which relies on applying wavelet transform on the reflected signals from the targets and then calculate the summation of power distribution at target locations which gave a high performance to differentiate between them. The significant difference between the distributions of the different target spectrum can be considered as a finger print for every target, also the multiple received reflections varied according target type.

We achieve a success to differentiate and to obtain Finger prints of almost targets by using illustrated technique. The finger prints of trusted simulation models were obtained to bring out the set of specific concepts in order to rely on them in real field measurements.

Data are obtained by using a GPR of pre-equipped test site. They were processed in order to reduce unnecessary signals in order to isolate signals for landmines. The finger prints of various buried targets were obtained by using illustrated technique too.

\section{Acknowledgement}

The authors like to express their thanks to Dr. Ahmed El Lithay for participation in the data collection. Also, thanks to our colleagues from the Electrical and Geothermal laboratory that helped us climes the data acquisition. Many thanks to Professor Sato, Tohoku University, Japan for his guidance and support.

\section{References}

1. Amazeen CA, Locke MC (1988) "Developmental status of the U.S. Army's new handheld standoff mine detection system (HSTAMIDS)," In: Proceedings of the 2nd International Conference on the Detection of Abandoned Land Mines 458: 193-197.

2. Amazeen CA, Locke MC (1996)“US Army's new handheld standoff mine detection system (HSTAMIDS)," In: Proceedings of the EUREL International Conference on The Detection of Abandoned Land Mines: A Humanitarian Imperative Seeking a Technical Solution 431: 172-176.

3. Sato M, Kobayashi T, Takahashi KJ, Fujiwara X Feng (2006) "Vehicle mounted SAR-GPR and its evaluation," in Detection and Remediation Technologies for Mines and Minelike Targets XI 6217.

4. Ho KC, Paul D Gader (2002) "A Linear Prediction Land Mine Detection Algorithm for Hand-Held Ground Penetrating Radar", IEEE Transactions on Geoscience And Remote Sensing 40: 1374-1384.

5. Gader PD, Nelson BN, Frigui H, Vaillette G, Keller JM (2000) "Fuzzy logic detection of land mines with ground penetrating radar", Signal Processing, Spec. Issue Fuzzy Logic Signal Processing 80: 1069-1084.

6. National Council of Human Rights (NCHR) (2005) Egypt and the problem of landmines, Background paper, The international confer ence for development and landmine clearance in the North West Coast, Cairo 27-29.

7. Mahmoud A. Mohana, Abbas Mohamed Abbas, Mohamed L Gomaa, Shereen M Ebrahim (2013) "Discrimination between Landmine and Mine-Like Targets Using Wavelets and Spectral Analysis", NRIAG Journal of Astronomy \& Geophysics 2: 54-66.

8. Mansour KHK (2012) "Subsurface Fracture Characterization Based on Polarimetric Borehole Radar and its Application to Estimation of Hydraulic Properties", PhD thesis, Tohoku University.

9. Abbas AM, Lethy AM Al (2005) "Implementation of Wave-Let Correlation to Locate Landmine-Like Objects Using GPR Data as Experimented on a Test Site", Journal of the college Petroleum Engineering and Mining. 